\title{
A Discretization of the Nonholonomic Chaplygin Sphere Problem ${ }^{\star}$
}

Yuri N. FEDOROV

Department de Matemática I, Universitat Politecnica de Catalunya, Barcelona, E-08028, Spain

E-mail: Yuri.Fedorov@upc.edu

URL: http://www.ma1.upc.edu/personal/fedorov.html

Received December 13, 2006, in final form February 26, 2007; Published online March 12, 2007

Original article is available at http://www.emis.de/journals/SIGMA/2007/044/

\begin{abstract}
The celebrated problem of a non-homogeneous sphere rolling over a horizontal plane was proved to be integrable and was reduced to quadratures by Chaplygin. Applying the formalism of variational integrators (discrete Lagrangian systems) with nonholonomic constraints and introducing suitable discrete constraints, we construct a discretization of the $n$-dimensional generalization of the Chaplygin sphere problem, which preserves the same first integrals as the continuous model, except the energy. We then study the discretization of the classical 3-dimensional problem for a class of special initial conditions, when an analog of the energy integral does exist and the corresponding map is given by an addition law on elliptic curves. The existence of the invariant measure in this case is also discussed.
\end{abstract}

Key words: nonholonomic systems; discretization; integrability

2000 Mathematics Subject Classification: 37J60; 37J35; 70H45

Dedicated to the memory of Vadim, who made me fascinated with discrete integrable systems

\section{Introduction}

The Chaplygin problem on a non-homogeneous sphere rolling over a horizontal plane without slipping is probably one of the best known integrable systems of the classical nonholonomic mechanics. Although being non-Hamiltonian in the whole phase space (see [2]), the equations of motion possess an invariant measure, which is a rather strong property putting them rather close to Hamiltonian systems.

It is even more interesting that the Chaplygin sphere appears to be an algebraic integrable system in the sense that generic level varieties of the first integrals are open subsets of 2-dimensional Abelian varieties and, after an appropriate time reparameterization, the phase flow becomes a straight line uniform on them, $[6,8]$.

Note that a Lax pair with a spectral parameter for the Chaplygin sphere, which could provide all the constants of motion, is still unknown and, probably does not exist. Hence, one cannot use the powerful method of Baker-Akhieser functions to find theta-function solution of the problem or to construct its integrable discretization by applying a Bäcklund transformation, as described in $[12,7]$, or by factorization of matrix polynomials.

Contents of the paper. We briefly recall the equations of motion of the Chaplygin sphere, as well as their $n$-dimensional generalization. Then we construct a discretization of the problem by applying the formalism of variational integrators with nonholonomic constraints recently developed in $[4,14]$. Apart from the nonholonomic distribution on the tangent bundle $T Q$ of

${ }^{\star}$ This paper is a contribution to the Vadim Kuznetsov Memorial Issue 'Integrable Systems and Related Topics'. The full collection is available at http://www.emis.de/journals/SIGMA/kuznetsov.html 
the configuration manifold $Q$, the formalism requires introducing discrete constraints on $Q \times Q$ which, in a certain sense, must be consistent with the continuous ones. As one can see in the literature $[4,10,15]$, the choice of discrete constraints is crucial for the behavior of the discretized nonholonomic system: the latter may inherit the main properties of its continuous counterpart, or may not. As an example, we note that although continuous systems with stationary nonholonomic constraints possess the energy integral, almost all their known discretizations do not enjoy this property (see e.g., [5]). Nevertheless, for a class of discretizations considered in [10] there exists a natural method to specify discrete constraints which ensures the exact preservation of energy.

Our choice of discrete nonholonomic constraints on $E(n) \times E(n)$ that mimics the condition of the sphere rolling without slipping over a horizontal plane allows us to construct a map discretizating the Chaplygin sphere problem, which has the form of a momentum conservation law and therefore preserves all the momenta integrals of the original system.

We then consider the discretization of the classical 3-dimensional problem for the case when the discrete angular momentum is vertical. In this special case the structure of the map is reminiscent to that of the Veselov-Moser discretization of the Euler top on $S O(3)[19,16]$ and an analog of the quadratic energy integral does exist.

This implies that the invariant manifolds of the discretization map are elliptic curves $\mathcal{E}$ and the map is described as an addition law. However, in contrast to most known integrable discrete systems, in the discrete Chaplygin sphere the translation on $\mathcal{E}$ depends not only on the constants of motion but also on the point on the curve. Thus, in order to find the explicit solution, we arrive at a rather difficult problem of reparameterization of $\mathcal{E}$ or its real part, which would make the translation constant. We notice that this problem is equivalent to the problem of the existence of an invariant measure of the map.

\section{The Chaplygin sphere and its multidimensional generalization}

Following Chaplygin [3], consider a dynamically non-symmetric sphere with inertia tensor $J$, radius $\rho$, and mass $m$ rolling without slipping over a horizontal plane. Assume that the mass center and the geometric center $C$ of the sphere coincide. Let $\gamma=\left(\gamma_{1}, \gamma_{2}, \gamma_{3}\right)^{T}$ be the vertical unit vector and $\omega=\left(\omega_{1}, \omega_{2}, \omega_{3}\right)^{T}, v \in \mathbb{R}^{3}$ be respectively the angular velocity of the sphere and the velocity of its center in the moving frame. The condition of non-slipping of the point $P$ of contact of the sphere with the horizontal plane is

$$
v+\rho \omega \times \gamma=0 .
$$

Here and below, $\times$ denotes the vector product in $\mathbb{R}^{3}$. On the configuration space of the problem, the Lie group $E(3)$, these equations define nonholonomic constraints: for any two positions of the sphere on the plane there exists a linking trajectory that satisfies (2.1).

Under these constraints the equations of motion can be reduced to the following closed system for $\omega, \gamma[3,9]^{1}$

$$
\Lambda \dot{\omega}=\Lambda \omega \times \omega+\frac{D}{F}\left\langle\Lambda^{-1} \gamma,(\Lambda \omega) \times \omega\right\rangle \gamma, \quad \dot{\gamma}=\gamma \times \omega,
$$

where

$$
D=m \rho^{2}, \quad \Lambda=J+D \mathbb{I}, \quad F=\left\langle J \gamma, \Lambda^{-1} \gamma\right\rangle \equiv 1-D\left\langle\gamma, \Lambda^{-1} \gamma\right\rangle,
$$

\footnotetext{
${ }^{1}$ We use our proper notation for the variables and parameters of the problem, which may differ from that used in the original paper [3].
} 
$\mathbb{I}$ being the identity $3 \times 3$ matrix. Let $K=\Lambda \omega-D\langle\omega, \gamma\rangle \gamma$ be the vector of the angular momentum of the sphere with respect to the contact point $P$. Then the system (2.2) also admits representation in form

$$
\dot{K}=K \times \omega, \quad \dot{\gamma}=\gamma \times \omega .
$$

Hence, like $\gamma$, the momentum $K$ is fixed in space. As a result, the system possesses four independent first integrals

$$
\langle\gamma, \gamma\rangle=1, \quad\langle K, \gamma\rangle \equiv\langle J \omega, \gamma\rangle=h, \quad\langle K, K\rangle=n, \quad\langle K, \omega\rangle=l .
$$

Here the last integral is the kinetic energy of the sphere and, since

$$
\Lambda \omega=K-\frac{D}{F}\left\langle\gamma, \Lambda^{-1} K\right\rangle \gamma
$$

it can be rewritten in terms of $K, \gamma$ :

$$
\left\langle K, \Lambda^{-1} K\right\rangle-\frac{D}{F}\left\langle\gamma, \Lambda^{-1} K\right\rangle^{2}
$$

In addition, the system (2.2) possesses an invariant measure

$$
\sqrt{\left\langle J \gamma, \Lambda^{-1} \gamma\right\rangle} d \omega_{1} \wedge \cdots \wedge d \gamma_{3}
$$

hence, by the Euler-Jacobi theorem (which is also often refereed to as the Jacobi last multiplier theorem, see e.g., [20]), it is integrable by quadratures and its generic invariant varieties are 2-dimensional tori.

There are two special types of the initial conditions, when the equations of motion are simplified. In the first case the momentum $K$ is horizontal, $\langle K, \gamma\rangle=0$. As shown by Chaplygin, after a time reparameterization and introducing spheroconic coordinates on $S^{2}=\langle\gamma, \gamma\rangle$ the variables separate and the system reduces to hyperelliptic quadratures. Theta function solution for the unreduced Chaplygin system in this, as well as in the generic case, was obtained in [8].

The second special case is described below.

The case of the vertical momentum $\boldsymbol{K}$. As noticed in [3] and as follows from the energy integral in (2.4), under the special initial conditions $K=h \gamma, h=$ const one has $\langle K, \omega\rangle=$ $h\langle\gamma, \omega\rangle=l$, i.e.,

$$
\langle\omega, \gamma\rangle=\frac{l}{h}=\text { const, } \quad \text { and } \quad \Lambda \omega=\frac{h^{2}+D l}{h} \gamma .
$$

As a result, the first vector equation in (2.2) takes the form of the Euler top equations

$$
\Lambda \dot{\omega}=\Lambda \omega \times \omega
$$

and integrals (2.4) reduce to

$$
\langle\omega, \Lambda \omega\rangle=l, \quad\langle\Lambda \omega, \Lambda \omega\rangle=k^{2}, \quad l, k=\text { const. }
$$

Hence, for almost all initial conditions $K=h \gamma$, the variables $\omega_{i}, \gamma_{i}$ are elliptic functions of the original time $t$ and the solution of the reduced system is periodic.

Multidimensional generalization. Now, following [9], consider the generalized Chaplygin problem on an $n$-dimensional ball rolling without slipping on an $(n-1)$-dimensional 'horizontal' hyperplane $H$ in $\mathbb{R}^{n}$. The configuration space for the ball is the Lie group $E(n)=(R, r)$, where 
$R \in S O(n), r \in \mathbb{R}^{n}$ are the rotation matrix of the sphere and the position vector of its center $C$.

For a trajectory $R(t), r(t)$, define the Lie algebra element $\left(\omega, v_{C}\right)$, where

$$
\omega=R^{-1} \dot{R} \in \operatorname{so}(n), \quad v_{C}=R^{-1} \dot{r} \in \mathbb{R}^{n}
$$

are respectively the angular velocity and the velocity of $C$ in the frame attached to the sphere.

Let now $\gamma \in \mathbb{R}^{n}$ be the unit vector orthogonal to the hyperplane $H$ and directed 'upwards', i.e., from $H$ to the center $C$, and, as above, $\rho$ be radius of the ball. Then the condition for the sphere rolling without slipping that generalizes (2.1) reads

$$
v_{C}+\rho \omega \gamma=0
$$

One can show that this vector constraint determines a non-integrable distribution on the tangent bundle $T E(n)$, which is neither left- no right-invariant with respect to the action of $E(n)$.

Next, introduce the angular momentum of the sphere in the body frame with respect to the center $C$,

$$
M=J \omega+\omega J \in s o^{*}(n),
$$

$J$ being a constant diagonal $n \times n$ matrix. Then, as shown in [9], the motion of the sphere is described by the following Euler-Lagrange equations

$$
\dot{M}+[\omega, M]=\rho F \wedge \gamma, \quad m\left(\dot{v}_{C}+\omega v_{C}\right)=F, \quad \dot{\gamma}+\omega \gamma=0,
$$

where $F \in \mathbb{R}^{n}$ is the reaction force acting at the point $P$ of contact of the sphere with the hyperplane $H$. Here all the vectors are considered in the frame attached to the ball.

Now, differentiating the constraints (2.9) and using the second equation in (2.11), we find $F=m \rho \dot{\omega} \gamma$. Then (2.11) gives

$$
\dot{M}+[\omega, M]=D(\dot{\omega} \Gamma+\Gamma \dot{\omega}), \quad \dot{\Gamma}+[\omega, \Gamma]=0,
$$

where $D=m \rho^{2}, \Gamma=\gamma \otimes \gamma \equiv \gamma \gamma^{T}$. Then this system can be represented in the following compact commutative form that generalizes (2.3)

$$
\dot{\mathbf{K}}+[\omega, \mathbf{K}]=0, \quad \dot{\Gamma}+[\omega, \Gamma]=0,
$$

where

$$
\mathbf{K}=J \omega+\omega J+D(\Gamma \omega+\omega \Gamma) \in s o^{*}(m)
$$

can be regarded as the angular momentum of the ball relative to the contact point $P$.

It is natural to introduce the nondegenerate inertia operator $\mathcal{A}: s o(n) \rightarrow \operatorname{so}(n)^{*}$ such that $\mathbf{K}=\mathcal{A} \omega$. Since $\mathcal{A}$ is nondegenerate, equations (2.12) give a closed system for the variables $\omega, \gamma$.

As follows from the form of (2.12), $\operatorname{Ad}_{R} \mathbf{K}$ is a constant tensor in the space. Hence this system has the following set of integrals

$$
\operatorname{tr} \mathbf{K}^{s}, \quad \operatorname{tr}\left(\mathbf{K}^{s} \Gamma^{l}\right), \quad \operatorname{tr} \Gamma=1, \quad s=2,4,6, \ldots, \quad l \in \mathbb{N} .
$$

naturally, the system also possesses the energy integral

$$
H=-\frac{1}{4} \operatorname{tr}(\omega \mathcal{A} \omega)
$$

and, as shown in [9], the invariant measure $\mu d \omega \wedge d \gamma$ with density $\mu=\sqrt{\operatorname{det} \mathcal{A}}$, which depends on the components of $\gamma$ only.

In the classical case $n=3$, under the isomorphism between $s o(3)$ and $\mathbb{R}^{3}$,

$$
\omega_{i j}=\varepsilon_{i j k} \omega_{k}, \quad \mathbf{K}_{i j}=\varepsilon_{i j k} K_{k}
$$

the integrals (2.14), (2.15) and the measure transform to (2.4) and (2.6) respectively.

Various properties of the Chaplygin sphere and its $n$-dimensional generalization were studied in $[6,11,8,18]$, although the integrability or nonintegrability of this generalization was not proved. 


\section{Discrete mechanical systems with nonholonomic constraints}

Before describing the discrete setting, let us recall that a continuous nonholonomic Lagrangian system is a triple $(Q, L, \mathcal{D})$, where $Q$ is a smooth $n$-dimensional configuration space, $L: T Q \rightarrow$ $\mathbb{R}$ is a smooth function called the Lagrangian, and $\mathcal{D} \subset T Q$ is a $k$-dimensional constraint distribution. Let $q=\left(q_{1}, \ldots, q_{n}\right)$ be local coordinates on $Q$. In the induced coordinates $(q, \dot{q})$ on the tangent bundle $T Q$ we write $L(q, \dot{q})$. It is assumed that the map

$$
\frac{\partial L}{\partial \dot{q}}: T Q \rightarrow T^{*} Q
$$

is invertible. A curve $q(t) \in Q$ is said to satisfy the constraints if $\dot{q}(t) \in \mathcal{D}_{q(t)}$ for all $t$. The equations of motion are given by the Lagrange-d'Alembert principle:

$$
\delta \int_{a}^{b} L\left(q^{i}, \dot{q}^{i}\right) d t=0
$$

where $\delta q(t) \in \mathcal{D}_{q(t)}$ for $t \in(a, b)$ and $\delta q(a)=\delta q(b)=0$. This principle is supplemented by the condition that the curve itself satisfies the constraints. Note that we take the variation before imposing the constraints.

Assuming that the constraint distribution is specified by a set of differential forms $A^{j}(q)$, $j=1, \ldots, s<n$,

$$
\mathcal{D}=\left\{\dot{q} \in T Q \mid\left\langle A^{j}(q), \dot{q}\right\rangle=0, j=1, \ldots, s\right\},
$$

the principle (3.1) implies the Euler-Lagrange equations with multipliers $\lambda_{j}$

$$
\frac{d}{d t} \frac{\partial L}{\partial \dot{q}}-\frac{\partial L}{\partial q}=\sum_{j=1}^{s} \lambda_{j} A^{j}(q)
$$

Coupled with (3.2), they give a complete description of the dynamics of the system.

Note that equations (3.3) conserve the energy

$$
E=\left\langle\frac{\partial L}{\partial \dot{q}}, \dot{q}\right\rangle-L
$$

The discrete Lagrange-d'Alembert principle and equations. Let, as above, $Q$ be a smooth manifold. According to [4], a discrete nonholonomic mechanical system on $Q$ is defined by three ingredients

(1) a discrete Lagrangian $\mathbb{L}: Q \times Q \rightarrow \mathbb{R}$;

(2) an $(n-s)$-dimensional distribution $\mathcal{D}$ on $T Q$ (given by equations (3.2));

(3) a discrete constraint manifold $\mathcal{D}_{d} \subset Q \times Q$, which has the same dimension as $\mathcal{D}$ and satisfies the condition $(q, q) \in \mathcal{D}_{d}$ for all $q \in Q$.

The dynamics is given by the following discrete Lagrange-d'Alembert principle (see [4]),

$$
\sum_{k=0}^{N-1}\left(D_{1} \mathbb{L}\left(q_{k}, q_{k+1}\right)+D_{2} \mathbb{L}\left(q_{k-1}, q_{k}\right)\right) \delta q_{k}=0, \quad \delta q_{k} \in \mathcal{D}_{q_{k}}, \quad\left(q_{k}, q_{k+1}\right) \in \mathcal{D}_{d}
$$

Here $D_{1} \mathbb{L}$ and $D_{2} \mathbb{L}$ denote the partial derivatives of the discrete Lagrangian with respect to the first and the second inputs, respectively.

The discrete constraint manifold is specified by the discrete constraint functions

$$
\mathcal{F}_{j}\left(q_{k}, q_{k+1}\right)=0, \quad j=1, \ldots, s,
$$

which impose the above restriction on the solution sequence $\left\{\left(q_{k}, q_{k+1}\right)\right\}$. 
Remark 1. If the discrete Lagrangian $\mathbb{L}$ is obtained from a continuous one, $L(q, \dot{q})$, via a discretization mapping $\Psi: Q \times Q \rightarrow T Q$ defined in a neighborhood of the diagonal of $Q \times Q$, i.e., $\mathbb{L}=L \circ \Psi$, then the variety $\mathcal{D}_{d}$ must be consistent with the continuous distribution $\mathcal{D}$. Namely, $\mathcal{D}_{d}$ is locally defined by the equations $A^{j} \circ \Psi=0, j=1, \ldots, s$.

In particular, if the distribution $\mathcal{D} \subset T Q$ is integrable, i.e., it defines an $(n-s)$-dimensional integral submanifold $\mathcal{N} \subset Q$, then $\mathcal{D}_{d} \subset Q \times Q$ is just the product $\mathcal{N} \times \mathcal{N}$.

We emphasize that, in general, the discretization mapping is not unique and hence there are many ways to define the discrete Lagrangian $\mathbb{L}$ and the discrete constraint manifold $\mathcal{D}_{d}$ for a given nonholonomic system $(Q, L, \mathcal{D}){ }^{2}$

The discrete principle implies that the solutions of a discrete nonholonomic system are represented by sequences $\left\{\left(q_{k}, q_{k+1}\right)\right\}$ that satisfy the discrete Lagrange-d'Alembert equations with multipliers

$$
D_{1} \mathbb{L}\left(q_{k}, q_{k+1}\right)+D_{2} \mathbb{L}\left(q_{k-1}, q_{k}\right)=\sum_{j=1}^{s} \lambda_{j}^{k} A_{j}\left(q_{k}\right) .
$$

The multipliers $\lambda_{j}^{k}$ are determined from the discrete constraints (3.4), but, in general, not uniquely. Hence, the map $Q \times Q \mapsto Q \times Q$ defined by (3.4), (3.5) is multi-valued.

Remark 2. According to [4], equations (3.5) introduce a well-defined mapping $\left(q_{k-1}, q_{k}\right) \mapsto$ $\left(q_{k}, q_{k+1}\right)$, if the $(n+s) \times(n+s)$ matrix

$$
\left(\begin{array}{cccc}
D_{1} D_{2} L_{d}\left(q_{k}, q_{k+1}\right) & A^{1}\left(q_{k}\right) & \cdots & A^{s}\left(q_{k}\right) \\
D_{2} \mathcal{F}_{1}\left(q_{k}, q_{k+1}\right) & 0 & \cdots & 0 \\
\vdots & \vdots & & \vdots \\
D_{2} \mathcal{F}_{s}\left(q_{k}, q_{k+1}\right) & 0 & \cdots & 0
\end{array}\right)
$$

is invertible for each $\left(q_{k}, q_{k+1}\right)$ in a neighborhood of the diagonal of $Q \times Q$.

\section{A discretization of the Chaplygin sphere}

Now we apply the above approach to discretize the generalized Chaplygin sphere problem on $E(n)$. The trajectory of such system is a sequence $\left(R_{k}, r_{k}\right), k \in \mathbb{Z}$. We choose the discrete Lagrangian in form

$$
\mathbb{L}=\frac{1}{2} \operatorname{Tr}\left(R_{k} J R_{k+1}^{T}\right)+\frac{m}{2}\left\langle\Delta r_{k}, \Delta r_{k}\right\rangle, \quad \Delta r_{k}=r_{k+1}-r_{k} .
$$

Here the rotational part $\frac{1}{2} \operatorname{Tr}\left(R_{k} J R_{k+1}^{T}\right)$ coincides with the Lagrangian of the Euler top on $S O(n)$ introduced in [19].

Note that, in view of (2.8), the continuous constraints (2.9) can be rewritten as

$$
\dot{r}+\rho \dot{R} R^{T} \vec{\gamma}=0,
$$

where $\vec{\gamma}$ is the unit normal vector in the fixed frame (without loss of generality we can set it to be $(0, \ldots, 0,1)^{T}$.)

Then, in view of (4.1), (4.2), in our case the discrete Lagrange-d'Alembert equations (3.5) take the form

$$
R_{k+1} J+R_{k-1} J=\Lambda_{k} R_{k}+\rho \vec{f}^{k} \vec{\gamma}^{T} R_{k},
$$

\footnotetext{
${ }^{2}$ An alternative approach to the discretization of nonholonomic systems based on a modification of canonical transformations was proposed in [14].
} 


$$
m\left(r_{k+1}-r_{k}\right)-m\left(r_{k}-r_{k-1}\right)=\vec{f}^{k}
$$

where $\Lambda_{k}$ is the symmetric matrix Lagrange multiplier and $\vec{f}^{k}=\left(f_{1}^{k}, \ldots, f_{n}^{k}\right)^{T}$, is the vector multiplier corresponding to the constraints (4.2).

In view of the property $R_{k} R_{k}^{T}=\mathbf{I}$, equations (4.3) yield

$$
\begin{aligned}
& R_{k+1} J R_{k}^{T}+R_{k-1} J R_{k}^{T}=\Lambda_{k}+\rho \vec{f}^{k} \vec{\gamma}^{T}, \\
& R_{k} J R_{k+1}^{T}+R_{k} J R_{k-1}^{T}=\Lambda_{k}^{T}+\rho \vec{\gamma} \vec{f}_{k}^{T} .
\end{aligned}
$$

Following [19], we introduce the discrete angular momentum of the sphere with respect to its center $C$ in space

$$
m_{k}=R_{k+1} J R_{k}^{T}-R_{k} J R_{k+1}^{T} \in s o(n) .
$$

Then (4.5), (4.4) give rise to

$$
m_{k}=m_{k-1}+\rho\left(\vec{f}^{k} \vec{\gamma}^{T}-\vec{\gamma} \vec{f}^{k}\right), \quad m\left(\Delta r_{k}-\Delta r_{k-1}\right)=\vec{f}^{k} .
$$

Next, like in [19], introduce the discrete momentum of the sphere in the body frame

$$
M_{k}=\operatorname{Ad}_{\mathrm{R}_{\mathrm{k}}} m_{k} \equiv \Omega_{k} J-J \Omega_{k}^{T}, \quad \Omega_{k}=R_{k}^{T} R_{k+1} \in S O(n),
$$

where $\Omega_{k}$ is the finite rotation in the body frame. In the continuous limit $\Omega_{k} \mapsto \mathbf{I}+\varepsilon \omega, \omega \in \operatorname{so}(n)$, it transforms to the momentum (2.10).

Then the system (4.6) gives

$$
\begin{aligned}
& M_{k}=\Omega_{k-1}^{T} M_{k-1} \Omega_{k-1}+\rho\left(R_{k}^{T} \vec{f}^{k} \gamma^{T} R_{k}-R_{k}^{T} \gamma_{k} \vec{f}^{T} R_{k}\right), \\
& m\left(\Delta r_{k}-\Delta r_{k-1}\right)=\vec{f}^{k}
\end{aligned}
$$

that is,

$$
M_{k}=\Omega_{k-1}^{T} M_{k-1} \Omega_{k-1}+\rho \vec{F}^{k} \wedge \gamma_{k}^{T}, \quad m\left(\Delta r_{k}-\Delta r_{k-1}\right)=\vec{f}^{k},
$$

where

$$
\vec{F}^{k}=R_{k}^{T} \vec{f}^{k}, \quad \gamma_{k}=R_{k}^{T} \vec{\gamma}
$$

represent the vectors $\vec{f}^{k}, \vec{\gamma}$ in the body frame. Equations (4.7) can be regarded as a discrete version of the equations of motion of the Chaplygin sphere (2.11).

Discrete constraints. In order to determine the vector multiplier $\vec{f}^{k}$ we must specify discrete constraints on $E(n) \times E(n)$. A naive choice that imitates the form of the continuous constraints $(2.9)$ is

$$
\Delta r_{k}+\rho \bar{\Omega}_{k} \bar{\gamma}=0
$$

where $\bar{\Omega}_{k}=R_{k+1} R_{k}^{T} \in S O(n)$ is the finite rotation of the sphere in the space frame. However, this choice allows that the displacement $\Delta r_{k}$ of the center $C$ may be not "horizontal" (i.e., orthogonal to the vertical vector $\bar{\gamma}$ ), which is incompatible with the mechanical setting of the problem.

For this reason, our choice of the corresponding discrete constraints (3.4) will be

$$
\Delta r_{k}+\frac{\rho}{2}\left(\bar{\Omega}_{k}-\bar{\Omega}_{k}^{T}\right) \vec{\gamma}=0
$$


which does ensure that $\Delta r_{k}$ is orthogonal to $\bar{\gamma}$. In the continuous limit $\bar{\Omega}_{k} \mapsto \mathbf{I}+\omega$ it transforms to the constraint (4.2).

Then, in view of (4.9) and the second equation in (4.7),

$$
\begin{aligned}
\vec{f}^{k} & =-\frac{m \rho}{2}\left(\bar{\Omega}_{k}-\bar{\Omega}_{k}^{T}\right) \vec{\gamma}+\frac{m \rho}{2}\left(\bar{\Omega}_{k-1}-\bar{\Omega}_{k-1}^{T}\right) \vec{\gamma}, \\
\vec{F}^{k} & =R_{k}^{T} \vec{f}^{k}=-\frac{m \rho}{2}\left(\Omega_{k}-\Omega_{k}^{T}\right) \gamma_{k}-\frac{m \rho}{2}\left(\Omega_{k-1}-\Omega_{k-1}^{T}\right) \gamma_{k},
\end{aligned}
$$

and, therefore,

$$
\begin{aligned}
\vec{F}^{k} \wedge \gamma_{k}^{T}= & -\frac{m \rho}{2}\left[\Omega_{k} \Gamma_{k}+\Gamma_{k} \Omega_{k}\right]+\frac{m \rho}{2}\left[\Omega_{k}^{T} \Gamma_{k}+\Gamma_{k} \Omega_{k}^{T}\right]+\frac{m \rho}{2}\left[\Omega_{k-1} \Gamma_{k}+\Gamma_{k} \Omega_{k-1}\right] \\
& -\frac{m \rho}{2}\left[\Omega_{k-1}^{T} \Gamma_{k}+\Gamma_{k} \Omega_{k-1}^{T}\right]
\end{aligned}
$$

where, as in Section $2, \Gamma_{k}=\gamma_{k} \otimes \gamma_{k} \equiv \gamma_{k} \gamma_{k}^{T}$.

As a result, we eliminate the multipliers $\vec{F}^{k}$ and from (4.7), (4.8) get the equations

$$
\begin{aligned}
M_{k} & +\frac{m \rho^{2}}{2}\left[\Omega_{k} \Gamma_{k}+\Gamma_{k} \Omega_{k}\right]-\frac{m \rho^{2}}{2}\left[\Omega_{k}^{T} \Gamma_{k}+\Gamma_{k} \Omega_{k}^{T}\right]=\Omega_{k-1}^{T} M_{k-1} \Omega_{k-1} \\
& +\frac{m \rho^{2}}{2}\left[\Omega_{k-1} \Gamma_{k}+\Gamma_{k} \Omega_{k-1}\right]-\frac{m \rho^{2}}{2}\left[\Omega_{k-1}^{T} \Gamma_{k}+\Gamma_{k} \Omega_{k-1}^{T}\right], \\
\gamma_{k} & =\Omega_{k-1}^{T} \gamma_{k-1},
\end{aligned}
$$

which define an implicit map $\mathcal{C}:\left(\Omega_{k-1}, \gamma_{k-1}\right) \mapsto\left(\Omega_{k}, \gamma_{k}\right)$.

Proposition 1. The map $\mathcal{C}$ admits the following compact representation

$$
\mathcal{K}_{k}=\Omega_{k-1}^{T} \mathcal{K}_{k-1} \Omega_{k-1}, \quad \Gamma_{k}=\Omega_{k-1}^{T} \Gamma_{k-1} \Omega_{k-1},
$$

where $\mathcal{K}_{k}$ is the discrete analog of the momentum with respect to the contact point $P$ of the sphere,

$$
\begin{aligned}
\mathcal{K}_{k} & =\Omega_{k}\left(J+\frac{D}{2} \Gamma_{k}\right)-\left(J+\frac{D}{2} \Gamma_{k}\right) \Omega_{k}^{T}+\frac{D}{2}\left(\Gamma_{k} \Omega_{k}-\Omega_{k}^{T} \Gamma_{k}\right) \\
& \equiv M_{k}+\frac{D}{2}\left(\Omega_{k} \Gamma_{k}-\Gamma_{k} \Omega_{k}^{T}\right)+\frac{D}{2}\left(\Gamma_{k} \Omega_{k}-\Omega_{k}^{T} \Gamma_{k}\right), \quad D=m \rho^{2} .
\end{aligned}
$$

Indeed, in the continuous limit $\Omega_{k} \mapsto \mathbf{I}+\varepsilon \omega, \varepsilon \ll 1$ the matrix $\mathcal{K} / \varepsilon$ tends precisely to the angular momentum (2.13) of the $n$-dimensional sphere with respect to its contact point, whereas the relations (4.12) transform to the continuous equations (2.12).

The map $\mathcal{C}$ therefore is evaluated as follows:

1) given $\Omega_{k-1}, \Gamma_{k-1}$, determine $\mathcal{K}_{k-1}$ by (4.13);

2) Calculate $\mathcal{K}_{k}, \Gamma_{k}$ by (4.12);

3) Given $\mathcal{K}_{k}, \Gamma_{k}$, reconstruct $\Omega_{k}$ as a solution of the matrix equation (4.13).

The latter equation, in general, has several solutions, hence the map $\mathcal{C}$ is multi-valued. An an immediate corollary of Proposition 1, we obtain

Proposition 2. Regardless to a branch of the map $\mathcal{C}$, it preserves the set of momenta integrals (2.14) of the continuous Chaplygin sphere problem with $\mathbf{K}$ replaced by $\mathcal{K}$. 
This property gives a solid justification of our choice of the discrete constraint (4.9). Note however that a discrete analog of the energy integral (2.15) in the generic case is unknown (see also Proposition 3 below).

Proof of Proposition 1. The second equation in (4.12) follows directly from (4.11). Now replacing $\Gamma_{k}$ in the right hand side of (4.10) by $\Omega_{k-1}^{T} \Gamma_{k-1} \Omega_{k-1}$ and using the identity $\Omega_{k-1} \Omega_{k-1}^{T}=\mathbf{I}$, we get

$$
\begin{aligned}
M_{k} & +\frac{m \rho^{2}}{2}\left[\Omega_{k} \Gamma_{k}+\Gamma_{k} \Omega_{k}\right]-\frac{m \rho^{2}}{2}\left[\Omega_{k}^{T} \Gamma_{k}+\Gamma_{k} \Omega_{k}^{T}\right]=\Omega_{k-1}^{T} M_{k-1} \Omega_{k-1} \\
& +\frac{m \rho^{2}}{2}\left[\Gamma_{k-1} \Omega_{k-1}+\Omega_{k-1}^{T} \Gamma_{k-1} \Omega_{k-1}^{2}\right]-\frac{m \rho^{2}}{2}\left[\left(\Omega_{k-1}^{T}\right)^{2} \Gamma_{k-1} \Omega_{k-1}+\Omega_{k-1}^{T} \Gamma_{k-1}\right],
\end{aligned}
$$

which, in view of (4.13), is equivalent to the first equation in (4.12).

Remark 3. The form of equations (4.12), (4.13) is reminiscent to that of the discrete Euler top on $S O(n)$ first described in [19],

$$
M_{k}=\Omega_{k-1}^{T} M_{k-1} \Omega_{k-1}, \quad M_{k}=\Omega_{k} J-J \Omega_{k}^{T},
$$

however, the discrete momentum $\mathcal{K}$ in (4.13) depends not only on $\Omega$, but also on $\gamma$. Note that, in view of Proposition 1 , the subsequent momenta $\mathcal{K}_{k-1}, \mathcal{K}_{k}$ admit curious intertwining relations

$$
\begin{aligned}
& \mathcal{K}_{k-1}=\Omega_{k-1}\left(J+\frac{D}{2}\left(\Gamma_{k-1}+\Gamma_{k}\right)\right)-\left(J+\frac{D}{2}\left(\Gamma_{k-1}+\Gamma_{k}\right)\right) \Omega_{k-1}^{T}, \\
& \mathcal{K}_{k}=\left(J+\frac{D}{2}\left(\Gamma_{k-1}+\Gamma_{k}\right)\right) \Omega_{k-1}-\Omega_{k-1}^{T}\left(J+\frac{D}{2}\left(\Gamma_{k-1}+\Gamma_{k}\right)\right),
\end{aligned}
$$

which are reminiscent to the following relation between the subsequent momenta of the discrete Euler top (4.14) (see [16])

$$
M_{k-1}=\Omega_{k-1} J-J \Omega_{k-1}^{T}, \quad M_{k}=J \Omega_{k-1}-\Omega_{k-1}^{T} J .
$$

\section{Discrete Chaplygin sphere on $E(3)$ and its particular solutions}

In the case $n=3$ we can make use of the following parameterization of the body finite rotations

$$
\Omega=\left(\begin{array}{ccc}
q_{0}^{2}+q_{1}^{2}-q_{2}^{2}-q_{3}^{2} & 2\left(q_{1} q_{2}-q_{3} q_{0}\right) & 2\left(q_{1} q_{3}+q_{2} q_{0}\right) \\
2\left(q_{1} q_{2}+q_{3} q_{0}\right) & q_{0}^{2}+q_{2}^{2}-q_{1}^{2}-q_{3}^{2} & 2\left(q_{2} q_{3}-q_{0} q_{1}\right) \\
2\left(q_{1} q_{3}-q_{2} q_{0}\right) & -2\left(q_{2} q_{3}-q_{0} q_{1}\right) & q_{0}^{2}+q_{3}^{2}-q_{1}^{2}-q_{2}^{2}
\end{array}\right),
$$

where $q_{0}, q_{i}$ are the Euler parameters subject to relations $q_{0}^{2}+q_{1}^{2}+q_{2}^{2}+q_{3}^{2}=1$ and $q_{0}>0$. The operator (5.1) describes a finite rotation in $\mathbb{R}^{3}$ about the vector $\mathbf{q}=\left(q_{1}, q_{2}, q_{3}\right)^{T}$ by the angle $\theta$ such that $q_{0}=\cos (\theta / 2)$ and $|\mathbf{q}|=\sin (\theta / 2)$ (see, e.g., [20]).

Let now $\vec{M}_{k}, \overrightarrow{\mathcal{K}}_{k} \in \mathbb{R}^{3}$ be the vector representations of $M_{k}, \mathcal{K}_{k} \in s o^{*}(3)$,

$$
\begin{aligned}
& \vec{M}=\left(M_{1}, M_{2}, M_{3}\right)^{T} \equiv\left(M_{32}, M_{13}, M_{21}\right)^{T}, \\
& \overrightarrow{\mathcal{K}}=\left(\mathcal{K}_{1}, \mathcal{K}_{2}, \mathcal{K}_{3}\right)^{T} \equiv\left(\mathcal{K}_{32}, \mathcal{K}_{13}, \mathcal{K}_{21}\right)^{T} .
\end{aligned}
$$

Then, in view of (4.13), we get simple expressions

$$
\vec{M}=2\left(\begin{array}{l}
\left(J_{2}+J_{3}\right) q_{0} q_{1}+\left(J_{2}-J_{3}\right) q_{2} q_{3} \\
\left(J_{1}+J_{3}\right) q_{0} q_{2}+\left(J_{3}-J_{1}\right) q_{1} q_{3} \\
\left(J_{1}+J_{2}\right) q_{0} q_{3}+\left(J_{1}-J_{2}\right) q_{1} q_{2}
\end{array}\right), \quad \overrightarrow{\mathcal{K}}=\vec{M}+2 D q_{0}(\mathbf{q}-\langle\gamma, \mathbf{q}\rangle \gamma)
$$


In the continuous limit, when the angle $\theta$ is small, $\theta=\varepsilon \omega, \varepsilon \ll 1$, we have $\mathbf{q}=\frac{\varepsilon}{2} \omega+O\left(\varepsilon^{3}\right)$, $q_{0}=1-O\left(\varepsilon^{2}\right)$, and, up to terms of order $\varepsilon$,

$$
\frac{1}{\varepsilon} \overrightarrow{\mathcal{K}}=\Lambda \omega-D\langle\gamma, \omega\rangle \gamma, \quad \Lambda=\operatorname{diag}\left(J_{2}+J_{3}+D, J_{1}+J_{3}+D, J_{1}+J_{2}+D\right),
$$

which coincides with the expression for the angular momentum vector $K$ in the classical Chaplygin problem.

As a result, in the vector form the map (4.12) reads

$$
\overrightarrow{\mathcal{K}}_{k}=\Omega_{k-1}^{T} \overrightarrow{\mathcal{K}}_{k-1}, \quad \gamma_{k}=\Omega_{k-1}^{T} \gamma_{k-1},
$$

where $\Omega_{k}$ is recovered from $\mathcal{K}_{k}, \gamma_{k}$ by solving (5.2) with respect to $\mathbf{q}$ and substituting the solution into (5.1). One can show that for real $\mathcal{K}, \gamma$, the equations (5.2) have at most 4 and at least 2 real solutions $\mathbf{q}$.

Like in most of other discrete systems, in order to choose one of the branches of the map (4.12) one should restrict to the case of sufficiently small finite rotations $\Omega_{k}$. In this case only one of the above real solutions $\mathbf{q}$ will be small and should be taken as the appropriate branch.

Like the continuous system (2.3), apart from the geometric condition $\langle\gamma, \gamma\rangle=1$, the discrete system preserves two independent integrals

$$
\langle\mathcal{K}, \gamma\rangle=h, \quad\langle\mathcal{K}, \mathcal{K}\rangle=n .
$$

However, as simple numerical tests show, the energy integral (2.5) is not preserved.

The special case $\overrightarrow{\mathcal{K}} \| \gamma$. Like the continuous Chaplygin system, the map (5.3) has the special case when $\overrightarrow{\mathcal{K}}_{k}=h \gamma_{k}, h=$ const. In view of (5.2), this implies the following relation between $\gamma$ and $\mathbf{q}$

$$
2\left(\begin{array}{c}
\left(\widehat{J}_{2}+\widehat{J}_{3}\right) q_{0} q_{1}+\left(\widehat{J}_{2}-\widehat{J}_{3}\right) q_{2} q_{3} \\
\left(\widehat{J}_{1}+\widehat{J}_{3}\right) q_{0} q_{2}+\left(\widehat{J}_{3}-\widehat{J}_{1}\right) q_{1} q_{3} \\
\left(\widehat{J}_{1}+\widehat{J}_{2}\right) q_{0} q_{3}+\left(\widehat{J}_{1}-\widehat{J}_{2}\right) q_{1} q_{2}
\end{array}\right)=\left[h+D q_{0}\langle\gamma, \mathbf{q}\rangle\right] \gamma, \quad \widehat{J}=J+\frac{D}{2} I .
$$

Then one obtains the reduced map $\mathcal{G}_{h}: S^{2} \mapsto S^{2}, \mathcal{G}_{h}\left(\gamma_{k-1}\right)=\gamma_{k}$ evaluated as follows:

1) given $\gamma_{k-1}$, one recovers $\mathbf{q}$ as a solution of (5.4),

2) one calculates $\Omega_{k}$ by (5.1) and $\gamma_{k}$ by the second equation in (5.3).

Proposition 3. For any branch of the map $\mathcal{G}_{h}$, it has the quadratic integral

$$
\left\langle\gamma, \Lambda^{-1} \gamma\right\rangle=l
$$

Proof. In view of (5.4), (5.1), and the second equation in $(5.3)^{3}$,

$$
\begin{aligned}
& \gamma_{k}=\frac{2}{\sigma_{k}}\left(\begin{array}{l}
\left(\widehat{J}_{2}+\widehat{J}_{3}\right) q_{0} q_{1}+\left(\widehat{J}_{2}-\widehat{J}_{3}\right) q_{2} q_{3} \\
\left(\widehat{J}_{1}+\widehat{J}_{3}\right) q_{0} q_{2}+\left(\widehat{J}_{3}-\widehat{J}_{1}\right) q_{1} q_{3} \\
\left(\widehat{J}_{1}+\widehat{J}_{2}\right) q_{0} q_{3}+\left(\widehat{J}_{1}-\widehat{J}_{2}\right) q_{1} q_{2}
\end{array}\right), \quad \sigma_{k}=\left[h+D\left\langle\gamma_{k}, q_{0} \mathbf{q}\right\rangle\right], \\
& \gamma_{k+1}=-\Omega_{k}^{T} \gamma_{k} \equiv \frac{2}{\sigma_{k}}\left(\begin{array}{l}
\left(\widehat{J}_{2}+\widehat{J}_{3}\right) q_{0} q_{1}-\left(\widehat{J}_{2}-\widehat{J}_{3}\right) q_{2} q_{3} \\
\left(\widehat{J}_{1}+\widehat{J}_{3}\right) q_{0} q_{2}-\left(\widehat{J}_{3}-\widehat{J}_{1}\right) q_{1} q_{3} \\
\left(\widehat{J}_{1}+\widehat{J}_{2}\right) q_{0} q_{3}-\left(\widehat{J}_{1}-\widehat{J}_{2}\right) q_{1} q_{2}
\end{array}\right) .
\end{aligned}
$$

Substituting these expressions into (5.5), we obtain $\left\langle\gamma_{k}, \Lambda^{-1} \gamma_{k}\right\rangle=\left\langle\gamma_{k+1}, \Lambda^{-1} \gamma_{k+1}\right\rangle$.

\footnotetext{
${ }^{3}$ To simplify notation, here and below we omit the discrete time index $k$ in the components of $\mathbf{q}$.
} 
As a result, in our special case the complex invariant variety of the map $\mathcal{G}_{h}$ is the spatial elliptic curve $\mathcal{E}$, the intersection of the unit sphere $\langle\gamma, \gamma\rangle=1$ with the quadric given by (5.5). The curve $\mathcal{E}$ is 4 -fold unramified covering of the planar elliptic curve

$$
\mathcal{E}_{0}=\left\{w^{2}=-\left(z-\Lambda_{1}^{-1}\right)\left(z-\Lambda_{2}^{-1}\right)\left(z-\Lambda_{3}^{-1}\right)(z-l)\right\}
$$

and the points of $\mathcal{E}$ are parameterized by the Jacobi elliptic functions associated to $\mathcal{E}_{0}$. Assume for concreteness that $\Lambda_{1}^{-1}<\Lambda_{2}^{-1}<l<\Lambda_{3}^{-1}$. Then the parameterization reads (see e.g., [20, 13])

$$
\begin{aligned}
& \gamma_{1}=C_{1} \operatorname{cn}(u \mid k), \quad \gamma_{2}=C_{2} \operatorname{sn}(u \mid k), \quad \gamma_{3}=C_{3} \operatorname{dn}(u \mid k), \\
& C_{1}=\sqrt{\frac{\Lambda_{1}\left(1-\Lambda_{3} l\right)}{\Lambda_{1}-\Lambda_{3}}}, \quad C_{2}=\sqrt{\frac{\Lambda_{2}\left(1-\Lambda_{3} l\right)}{\Lambda_{2}-\Lambda_{3}}}, \quad C_{3}=\sqrt{\frac{\Lambda_{3}\left(1-\Lambda_{1} l\right)}{\Lambda_{1}-\Lambda_{3}}},
\end{aligned}
$$

where $u$ is a complex phase parameter and $k$ is the modulus of $\mathcal{E}_{0}$ given by

$$
k^{2}=\frac{\left(\Lambda_{1}-\Lambda_{2}\right)\left(1-\Lambda_{3} l\right)}{\left(\Lambda_{2}-\Lambda_{3}\right)\left(\Lambda_{1} l-1\right)} .
$$

Therefore, for a fixed $l$, the map $\mathcal{G}_{h}$ is reduced to one-dimensional map

$$
u_{k+1}=u_{k}+\Delta u_{k}\left(u_{k}, l\right),
$$

with the increment $\Delta u_{k}$ is a function of $u_{k}, l$ to be determined below.

Remark 4. As seen from (5.6), the structure of the map $\mathcal{G}_{h}$ is similar to that of the VeselovMoser discretization (4.14) of the Euler top on so(3). As shown in [16, 1], the latter discretization preserves the momentum and the energy integrals, whereas the corresponding increment $\Delta u_{k}$ does not depend on the argument $u_{k}$ in the elliptic parameterization. The same holds for another exact discretization of the top found in [7].

In comparison with the Veselov-Moser discretization, our map $\mathcal{G}_{h}$ involves the extra factor $\sigma_{k}$, which is not constant on the orbits of $\mathcal{G}_{h}$. This gives an indication that under our map $\mathcal{G}_{h}$ the increment $\Delta u_{k}$ depends essentially on $u_{k}$. Moreover, as $\mathcal{G}_{h}$ is multi-valued, the function $\Delta u_{k}\left(u_{k}, l\right)$ is expected to be multi-valued as well. This stays in contrast with the solutions of the continuous equations (2.7) obtained from the Chaplygin sphere system under the condition $K \| \gamma$ : the components of $\gamma$ are elliptic functions whose argument changes uniformly with time $t$.

In this connection the following natural problem of the complete integrability of the map $\mathcal{G}_{h}$ arises: is there a (multi-valued) reparameterization $u=f(s, l), s \in \mathbb{C}$ such that

$$
u_{k}=f\left(s_{k}\right), \quad u_{k+1}=f\left(s_{k}+\Delta s\right)
$$

and the increment $\Delta s$ does not depend on $s_{k}$ ? Note that this property is equivalent to that the one-dimensional map (5.9) preserves the invariant measure $\mu(u) d u$, where the density $\mu(u)$ must satisfy the discrete Liouville equation

$$
\frac{\partial u_{k+1}}{\partial u_{k}}=\frac{\mu\left(u_{k}\right)}{\mu\left(u_{k+1}\right)}
$$

and is related to $f(s)$ by the formula $d f / d s=1 / \mu$. Due to the existence of the integral (5.5), this is also equivalent to that the map $\mathcal{G}_{h}$ itself preserves an invariant measure on $S^{2}$.

Since $u$ is the argument of an elliptic function, the restriction of the map (5.9) onto the real axis describes a diffeomorphism of a circle with the angular function $\Delta u_{k}\left(u_{k}, l\right)$. Hence, the answer to the above integrability question requires studying the properties of the diffeomorphism and applying known theorems in this field (see, e.g., [17] and references therein).

Determination of $\Delta u_{k}\left(u_{k}, l\right)$. For convenience, in the sequel we omit the discrete time index $k$ and denote $\mathcal{G}_{h}(\gamma)=\tilde{\gamma}, u+\Delta u=\tilde{u}$. To determine the function $\Delta u(u, l)$, we shall use the following property. 
Proposition 4. The map $\mathcal{G}_{h}$ admits the following implicit form that involves the sum and the difference of $\gamma, \tilde{\gamma}$ only:

$$
\begin{aligned}
& \tilde{\gamma}-\gamma=\frac{\varkappa}{4}(\tilde{\gamma}+\gamma) \times \Lambda^{-1}(\tilde{\gamma}+\gamma), \\
& \varkappa=\frac{2 \sigma}{1+\sqrt{1-\frac{1}{4}\left\langle\tilde{\gamma}+\gamma, \Lambda^{-2}(\tilde{\gamma}+\gamma)\right\rangle}}, \\
& \sigma=\frac{h}{1-\frac{D}{8}\left\langle\tilde{\gamma}+\gamma, \Lambda^{-1}(\tilde{\gamma}+\gamma)\right\rangle} .
\end{aligned}
$$

Proof. As follows from (5.6),

$$
\tilde{\gamma}+\gamma=\frac{4 q_{0}}{\sigma} \Lambda \mathbf{q}, \quad \tilde{\gamma}-\gamma=\frac{4}{\sigma} \Lambda \mathbf{q} \times \mathbf{q}
$$

and, therefore,

$$
\tilde{\gamma}-\gamma=\frac{\sigma}{4 q_{0}^{2}}(\tilde{\gamma}+\gamma) \times \Lambda^{-1}(\tilde{\gamma}+\gamma)
$$

Using (5.12), it is easy to check that

$$
2 q_{0}^{2}=1+\sqrt{1-\frac{1}{4}\left\langle\Lambda^{-1}(\tilde{\gamma}+\gamma), \Lambda^{-1}(\tilde{\gamma}+\gamma)\right\rangle}
$$

and that the factor $\sigma$ defined in (5.6) is the solution of the equation

$$
\sigma=h+D\left\langle\gamma, \frac{\sigma}{4} \Lambda^{-1}(\tilde{\gamma}+\gamma)\right\rangle
$$

Then, in view of relation

$$
\left\langle\gamma, \Lambda^{-1}(\tilde{\gamma}+\gamma)\right\rangle=\frac{1}{2}\left\langle\gamma+\tilde{\gamma}, \Lambda^{-1}(\gamma+\tilde{\gamma})\right\rangle
$$

one obtains (5.10), (5.11).

Below we assume $h=1$ in (5.11), which can always be made by appropriate rescaling of $\Lambda$ and $D$.

Implicit maps in the symmetric form (5.10) were studied in [1] as special discretizations of the Euler top on $s o(3)$. The symmetry allows to determine the function $\Delta u(u, l)$ in $(5.9)$. Following [1], we set

$$
\bar{u}=\frac{u+\tilde{u}}{2}, \quad \delta u=\frac{\tilde{u}-u}{2} .
$$

Then, due to addition formulas for the Jacobi elliptic functions (see e.g., [13]), the parameterization (5.7), (5.8) implies

$$
\begin{aligned}
& \tilde{\gamma}_{1}-\gamma_{1}=\frac{2 C_{1}}{\mathcal{D}} \operatorname{sn}(\bar{u}) \operatorname{dn}(\bar{u}) \operatorname{sn}(\delta u) \operatorname{dn}(\delta u), \quad \tilde{\gamma}_{1}+\gamma_{1}=\frac{2 C_{1}}{\mathcal{D}} \operatorname{cn}(\bar{u}) \operatorname{cn}(\delta u), \\
& \mathcal{D}=1-k^{2} \operatorname{sn}^{2}(\bar{u}) \operatorname{sn}^{2}(\delta u)
\end{aligned}
$$

and similar expressions for the other components of $\tilde{\gamma}-\gamma, \tilde{\gamma}+\gamma$. Substituting them into any of the equations (5.10), one obtains (see also [1])

$$
\frac{2 \mathcal{D}}{\varkappa}=V \frac{\operatorname{cn}(\delta u) \operatorname{dn}(\delta u)}{\operatorname{sn}(\delta u)}, \quad V^{2}=\frac{\left(\Lambda_{2}-\Lambda_{3}\right)\left(\Lambda_{1} l-1\right)}{\Lambda_{1} \Lambda_{2} \Lambda_{3}}
$$


or, in view of $(5.11)$,

$$
\begin{aligned}
& \left(1-\frac{d}{2} \Pi_{1}\right) \sqrt{\mathcal{D}^{2}-\Pi_{2} \mathcal{D}^{2}}=V \frac{\operatorname{cn}(\delta u) \operatorname{dn}(\delta u)}{\operatorname{sn}(\delta u)}-\mathcal{D}\left(1-\frac{d}{2} \Pi_{1}\right), \\
& \Pi_{1}=\frac{1}{4}\left\langle\tilde{\gamma}+\gamma, \Lambda^{-1}(\tilde{\gamma}+\gamma)\right\rangle, \quad \Pi_{2}=\frac{1}{4}\left\langle\tilde{\gamma}+\gamma, \Lambda^{-2}(\tilde{\gamma}+\gamma)\right\rangle .
\end{aligned}
$$

Then, making squares of both parts of (5.14) and taking into account the expressions

$$
\begin{aligned}
\Pi_{1} & =\frac{1}{\mathcal{D}}\left(\frac{C_{1}^{2}}{\Lambda_{1}}+\frac{C_{3}^{2}}{\Lambda_{3}}-\frac{C_{2}^{2}}{\Lambda_{2}} \operatorname{sn}^{2}(\delta u)\right) \equiv \frac{1}{\mathcal{D}}\left(l-\mu \operatorname{sn}^{2}(\delta u)\right), \quad \mu=\frac{1-\Lambda_{3} l}{\Lambda_{2}-\Lambda_{3}}, \\
\Pi_{2} & =\frac{1}{\mathcal{D}^{2}}\left(\frac{C_{1}^{2}}{\Lambda_{1}^{2}} \operatorname{cn}^{2}(\bar{u}) \operatorname{cn}^{2}(\delta u)+\frac{C_{2}^{2}}{\Lambda_{2}^{2}} \operatorname{sn}^{2}(\bar{u}) \operatorname{cn}^{2}(\delta u) \operatorname{dn}^{2}(\delta u)+\frac{C_{3}^{2}}{\Lambda_{3}^{2}} \operatorname{dn}^{2}(\bar{u}) \operatorname{dn}^{2}(\delta u)\right) \\
& \equiv \frac{1}{\mathcal{D}^{2}}\left(\frac{G}{\operatorname{sn}^{2}(\delta u)} \mathcal{D}-V^{2} \frac{\operatorname{cn}^{2}(\delta u) \operatorname{dn}^{2}(\delta u)}{\operatorname{sn}^{2}(\delta u)}\right),
\end{aligned}
$$

where

$$
\begin{aligned}
& G=\frac{C_{1}^{2}}{k^{2} \Lambda_{1}^{2}} \operatorname{cn}^{2}(\delta u)-\frac{C_{2}^{2}}{\Lambda_{2}^{2}} \operatorname{cn}^{2}(\delta u) \operatorname{dn}^{2}(\delta u)+\frac{C_{3}^{2}}{\Lambda_{3}^{2}} \operatorname{dn}^{2}(\delta u) \equiv V^{2}\left(1+\alpha \operatorname{sn}^{2}(\delta u)-\beta \operatorname{sn}^{4}(\delta u)\right), \\
& \alpha=\frac{2 \Lambda_{1} \Lambda_{3}-\left(\Lambda_{1}+\Lambda_{3}-\Lambda_{2}\right)}{\left(\Lambda_{2}-\Lambda_{3}\right)\left(\Lambda_{1} l-1\right)}, \quad \beta=\frac{\Lambda_{1} \Lambda_{3}}{\left(\Lambda_{2}-\Lambda_{3}\right)^{2}} \frac{1-\Lambda_{3} l}{\Lambda_{1} l-1},
\end{aligned}
$$

after simplifications we obtain the following relation between the Jacobi elliptic functions of $\delta u$ and the function $\operatorname{sn}(\bar{u})$ (contained in $\mathcal{D}$ only)

$$
\begin{aligned}
& -\frac{d^{2}}{4} \frac{\left(l-\mu \operatorname{sn}^{2}(\delta u)\right)^{2}}{\mathcal{D}^{2}}\left(G \mathcal{D}-V^{2} \operatorname{cn}^{2}(\delta u) \operatorname{dn}^{2}(\delta u)\right) \\
& \quad+d \frac{l-\mu \operatorname{sn}^{2}(\delta u)}{\mathcal{D}}\left(\left(G \mathcal{D}-V^{2} \operatorname{cn}^{2}(\delta u) \operatorname{dn}^{2}(\delta u)\right)-V w(\delta u) \mathcal{D}\right)+\mathcal{D}(2 V w(\delta u)-G)=0, \\
& w(\delta u)=\operatorname{sn}(\delta u) \operatorname{cn}(\delta u) \operatorname{dn}(\delta u) .
\end{aligned}
$$

Note that in the case $d=0$, when the map $\mathcal{G}_{h}$ reduces to the discretization of the Euler top, the terms with $\mathcal{D}$ in (5.15) can be eliminated and the increment $\delta u$ becomes independent of $\bar{u}$, as expected and was shown explicitly in [1].

Now, expressing $\operatorname{sn}(\bar{u}) \equiv \operatorname{sn}(u+\delta u)$ in terms of elliptic functions of $u$ and $\delta u$, and using relations $\operatorname{cn}(u)^{2}=1-\operatorname{sn}^{2}(u), \operatorname{dn}(u)^{2}=1-k^{2} \operatorname{sn}^{2}(u)$, we can rewrite (5.13) as

$$
\begin{aligned}
& \mathcal{D}=\frac{1}{\left[1-k^{2} \operatorname{sn}^{2}(u) \operatorname{sn}^{2}(\delta u)\right]^{2}}\left(k^{2} \operatorname{sn}^{2}(u) \operatorname{sn}^{2}(\delta u)\left(2-\left(k^{2}+1\right) \operatorname{sn}^{2}(\delta u)-k^{2} \operatorname{sn}^{4}(\delta u)\right)\right. \\
& \left.\quad+2 k^{2} \operatorname{sn}^{2}(\delta u) w(\delta u) W(u)+k^{2} \operatorname{sn}^{4}(\delta u)\left[1-k^{2} \operatorname{sn}^{2}(u)\right]-1\right), \\
& W(u)=\operatorname{sn}(u) \operatorname{cn}(u) \operatorname{dn}(u), \quad w(\delta u)=\operatorname{sn}(\delta u) \operatorname{cn}(\delta u) \operatorname{dn}(\delta u) .
\end{aligned}
$$

Substituting this expression into (5.15), one obtains a rather complicated equation for $\mathrm{sn}^{2}(u)$, $\operatorname{sn}^{2}(\delta u), w(\delta u)$ that implicitly describes the dependence the function $\Delta u_{k}\left(u_{k}, l\right)$.

One can make this equation algebraic by introducing the variables $p=\wp(\delta u), q=\wp^{\prime}(\delta u)$, which are coordinates on the curve $\mathcal{E}$ represented in the canonical Weierstrass form

$$
q^{2}=4\left(p-e_{1}\right)\left(p-e_{2}\right)\left(p-e_{3}\right), \quad e_{1}=\frac{1}{3}\left(2-k^{2}\right), \quad e_{2}=\frac{1}{3}\left(2 k^{2}-1\right), \quad e_{3}=-\frac{1}{3}\left(1+k^{2}\right) .
$$

Under the rational parameterization

$$
\operatorname{sn}^{2}(\delta u)=\frac{1}{p-e_{3}}, \quad \operatorname{cn}^{2}(\delta u)=\frac{p-e_{1}}{p-e_{3}}, \quad \operatorname{dn}^{2}(\delta u)=\frac{p-e_{2}}{p-e_{3}}, \quad w\left(\delta_{u}\right)=\frac{1}{2} \frac{q}{\left(p-e_{3}\right)^{3}}
$$


equation (5.15) together with (5.16) defines a plane algebraic curve whose intersections $P^{(j)}$ with $\mathcal{E}_{0}$ give the values of $\delta u$ via the integral

$$
\delta u^{(j)}=\int_{\infty}^{P^{(j)}} \frac{d p}{2 \sqrt{\left(p-e_{1}\right)\left(p-e_{2}\right)\left(p-e_{3}\right)}} .
$$

\section{Conclusive remarks}

In given paper we constructed a discretization of the Chaplygin sphere problem on $E(n)$ which preserves all the momenta integrals and, in the particular case of the motion, the energy. This case is reduced to the multi-valued map $\mathcal{G}_{h}$ on $s o^{*}(3)$ which is reminiscent to the Veselov-Moser discretization of the Euler top and is given by an addition law on elliptic curves. However, in contrast to known integrable maps of this type, the increment depends not only on the energy constant, but also on the point on the curve. According to [1], this implies that $\mathcal{G}_{h}$ does not preserve the standard Lie-Poisson structure on $s o^{*}(3)$, which is quite expected due to the nonholonomic origin of the map.

The integrability of the map $\mathcal{G}_{h}$ (which is interpreted as the possibility of writing its explicit discrete time solution) was shown to be equivalent to preservation of an invariant measure on the Lie algebra. Note that the latter property is often related to integrability in quadratures in the continuous case (recall the Jacobi last multiplier theorem).

In this connection it would be interesting to develop integrability criteria for discrete systems preserving an invariant measure, in particular to formulate a discrete version of the Jacobi last multiplier theorem.

Although the energy (2.5) of the Chaplygin sphere expressed in terms of the momentum $\mathcal{K}$ is not preserved in the discrete setting in general, this does not exclude the existence of another integral that transforms to the energy in the continuous limit.

Lastly, as shown in [18], the classical Chaplygin sphere problem is a natural example of a nonholonomic LR system on the direct product $S O(3) \times \mathbb{R}^{3}$ (the kinetic energy is left-invariant, while the constraint distribution is right-invariant with respect to the group action). One can show that the discrete Lagrangian (4.1) and the discrete constraint subvariety given by (4.9) possess the same properties, i.e., our discretization of the Chaplygin sphere is a discrete LR system on $S O(n) \times \mathbb{R}^{n}$.

We then believe that it is worth studying properties of generic discrete LR systems and of their reductions, such as preservation of momenta, energy, and an invariant measure.

\section{Acknowledgments}

I am grateful to the anonymous referees whose remarks helped to improve the text. The research was partially supported by Spanish Ministry of Science and Technology grant BFM 2003-09504C02-02.

\section{References}

[1] Bobenko A.I., Lorbeer B., Suris Yu., Integrable discretizations of the Euler top, J. Math. Phys. 39 (1998), 6668-6683, solv-int/9803016.

[2] Borisov A., Mamaev I., An obstruction to nonholonomic systems being Hamiltonian, Dokl. Akad. Nauk 387 (2002), 764-766 (English transl.: Dokl. Phys. 47 (2002), 892-894).

[3] Chaplygin S.A., On rolling of a ball on a horisontal plane, in Collection of Works, Vol. 1, Nauka, Moscow, 1981 (English transl.: Regul. Chaotic Dyn. 7 (2002), 131-148).

[4] Cortés J., Martínez S., Nonholonomic integrators, Nonlinearity 14 (2001), 1365-1392. 
[5] Cortés J., Energy-conserving nonholonomic integrators, Discrete Contin. Dyn. Syst. (2003), suppl., 189-199, math.NA/0209314.

[6] Duistermaat J., Chaplygin's sphere, math.DS/0409019.

[7] Fedorov Yu., Integrable flows and Bäcklund transformations on extended Stiefel varieties with application to the Euler top on the Lie group SO(3), J. Nonlinear Math. Phys. 12 (2005), suppl. 2, 77-94, nlin.SI/0505045.

[8] Fedorov Yu., A complete complex solution of the nonholonomic Chaplygin sphere problem, Preprint.

[9] Fedorov Yu., Kozlov V., Various aspects of $n$-dimensional rigid body dynamics, Amer. Math. Soc. Transl. Ser. 2168 (1995), 141-171.

[10] Fedorov Yu., Zenkov D., Discrete nonholonomic LL systems on Lie groups, Nonlinearity 18 (2005), 22112241, math.DS/0409415.

[11] Kilin A., The dynamics of Chaplygin ball: the qualitative and computer analysis, Regul. Chaotic Dyn. 6 (2001), 291-306.

[12] Kuznetsov V., Vanhaecke P., Bäcklund transformation for finite-dimensional integrable systems. A geometric approach, J. Geom. Phys. 44 (2002), 1-40, nlin.SI/0004003.

[13] Lawden D., Elliptic functions and applications, Springer-Verlag, 1989.

[14] de León M., Martín de Diego D., Santamaría Merino A., Geometric integrators and nonholonomic mechanics, J. Math. Phys. 45 (2004), 1042-1062.

[15] McLachlan R., Perlmutter M., Integrators for nonholonomic mechanical systems, J. Nonlinear Sci. 16 (2006), 283-328.

[16] Moser J., Veselov A., Discrete versions of some classical integrable systems and factorization of matrix polynomials, Comm. Math. Phys. 139 (1991), 217-243.

[17] Pérez-Marco R., Fixed points and circle maps, Acta Math. 179 (1997), 243-294.

[18] Schneider D., Non-holonomic Euler-Poincaré equations and stability in Chaplygin's sphere, Dyn. Syst. 17 (2002), 87-130.

[19] Veselov A. Integrable discrete-time systems and difference operators, Funct. Anal. Appl. 22 (1988), 83-94.

[20] Whittaker E.T., A treatise on analytical dynamics, 4th ed., Cambridge Univ. Press, Cambridge, 1960. 